\title{
PREVALENCIA DE ADENOCARCINOMA PROSTÁTICO INCIDENTAL TRAS ADENOMECTOMIAA SUPRAPÚBICA CON O SIN BIOPSIA PROSTÁTICA PREVIA.
}

\author{
Enrique Fernández Rosado, Francisco Gómez Veiga, Luis Álvarez Castelo, Manuel Ruibal \\ Moldes, Juan Manuel Mosquera Reboredo' y Marcelino González Martin.
}

Servicio de Urología. Servicio de Anatomía Patológica'. Complejo Hospitalario Universitario Juan Canalejo. La Coruña. España.

\begin{abstract}
Resumen.- OBJETIVO: Determinar la prevalencia de cáncer de próstata incidental tras biopsia prostática transrectal ecodirigida y posterior adenomectomía prostática suprapúbica, y compararlo con la prevalencia de un grupo similar de pacientes que no fueron biopsiados previamente a la cirugía. Evaluar el tratamiento, la evolución y las progresiones de la enfermedad tumoral en los pacientes con cáncer de próstata incidental.

MÉTODOS: Estudio retrospectivo de 549 adenomectomías suprapúbicas realizadas entre 1996-200116 años), comparando el grupo de pacientes biopsiados previamente a la adenomectomía con el grupo no biopsiado.
\end{abstract}

RESULTADOS: 291 (53\%) pacientes no fueron biopsiados previamente a la adenomectomía. 258 (47\%) fue- ron biopsiados. 25 cánceres de próstata incidentales detectados, 19 (76\%) en el grupo de los no biopsiados y $6(24 \%)$ en el de los biopsiados. $88 \%$ pTla y $12 \%$ pT1b. Gleason medio 4,5 (3-7). No fueron tratados el $84 \%$ de los pacientes (21) ("esperar y ver"); bloqueo hormonal 8\% (2); finasteride 8\% (2). Progresaron 3 pacientes (12\%), todos del grupo de los no tratados. Media de seguimiento de 48,1 meses (22-96). No se detectó ningún caso de mortalidad cáncer de próstata específico.

CONCLUSIONES: La prevalencia global de cáncer de próstata incidental en nuestra serie en pacientes a los que se les realizo adenomectomía prostática suprapúbica fue del 4,55\%. La prevalencia fue mayor en el grupo de pacientes no biopsiados previamente a la adenomectomía (3,46\%) que en el grupo de los biopsiados (1,09\%). La progresión tumoral fue del $12 \%$ y la supervivencia cáncer de próstata específica del 100\% tras una media de seguimiento de 48,1 meses (22-92). La biopsia prostática previa a la adenomectomía en pacientes con tacto rectal sospechoso o PSA elevado disminuye la prevalencia de cáncer prostático incidental. El seguimiento expectante activo puede ser una actitud valida en determinados casos.

Palabras clave: Cáncer próstata incidental. Adenomectomía prostática. Biopsia prostática.

Summary.- OBJECTIVES: To establish the prevalence of incidental prostate cancer after transrectal ultrasound guided prostatic biopsy and subsequent suprapubic prostatic adenomectomy and to compare it with a similar group of patients who did not underwent biopsy before surgery. To evaluate treatment, outcomes, and disease progression in patients with incidental prostate cancer. 
METHODS: Retrospective study of 549 suprapubic adenomectomy performed between 1996-200 1 (6 yr.), comparing the group of patients with biopsies before surgery vs. the group of patients without biopsies.

RESULTS: 291 (53\%) patients did not undergo biopsy before adenomectomy. 258 (47\%) underwent biopsies. 25 incidental prostate cancers were detected, 19 (76\%) in the group of no biopsy and $6(24 \%)$ in the biopsy group. $88 \% \mathrm{pT} 1 \mathrm{a}$ and $12 \% \mathrm{pT} 1 \mathrm{~b}$. Mean Gleason score 4.5 (3-7). $84 \%$ of the patients did not receive treatment (21) ("wait and see"); 8\% (2) androgen blockade; 8\% (2) finasteride (2). Three patients (12\%) in the group of no biopsy had disease progression. Mean follow-up was 48.1 months (22-96). No case of cancer-specific mortality was detected.

CONCLUSIONS: Global prevalence of incidental prostate cancer in our series of patients undergoing suprapubic prostatic adenomectomy was $4.55 \%$. Prevalence was higher in the group of patients without previous biopsy (3.46\%) than in the biopsy group (1.09\%). Tumor progression was $12 \%$ and cancer specific survival $100 \%$ after a mean follow-up of 48.1 months (22-92).

Previous prostatic biopsy in patients with suspicions digital rectal examination or elevated PSA diminishes the prevalence of incidental prostate cancer. Watchful waiting may be a valid option in some cases.

Keywords: Incidental prostate cancer. Prostatic adenomectomy. Prostatic biopsy.

\section{INTRODUCCIÓN}

La prostatectomía abierta o más correctamente denominada Adenomectomía Prostática Suprapúbica (APS) está actualmente considerada como uno de los tratamientos de la Hiperplasia Benigna de Próstata (HBP) sintomática que no ha respondido de modo totalmente satisfactorio al tratamiento farmacológico conservador, especialmente en próstatas de grandes volúmenes. La principal indicación de este procedimiento es el volumen de la glándula, considerando los $60 \mathrm{~cm}^{3}$ ecográficos como barrera que separa la cirugía endoscópica de la cirugía abierta. Este límite aunque es subjetivo y variable, depende en gran medida de la correcta y adecuada medición ecográfica de la glándula y de la experiencia y destreza en cirugía endoscópica del urólogo cirujano.
Pero no solo la cirugía abierta es volumen dependiente, sino que existen otras indicaciones establecidas para este tipo de cirugía independientemente del tamaño de la glándula como es la presencia de litiasis vesical grande, determinadas estenosis de uretra, divertículos vesicales que precisen ser resecados, problemas ortopédicos de los pacientes que imposibiliten la movilización de las piernas o la posición de litotomía, y otros menos frecuentes. La APS tiene un índice de reintervenciones $4-6$ veces menor $y$ una mortalidad mayor que el procedimiento endoscópico (1). A causa del perfeccionamiento de la técnica quirúrgica de la resección transuretral, el empleo de diversas técnicas, la destreza del urólogo cirujano y el diagnostico cada vez mas precoz de la HBP sintomática, aproximadamente el $90 \%$ de esta cirugía se realiza de modo endoscópico, dejando casos seleccionados para el procedimiento quirúrgico abierto.

Los pacientes a los que se les va a realizar esta intervención son sometidos o no previamente a una biopsia prostática como método de cribaje de patología tumoral prostática maligna. La indicación de dicha biopsia viene determinada por diversos parámetros y la interpretación que se haga de los mismos. Los principales parámetros son la edad del paciente, el examen prostático mediante tacto dígito rectal, el volumen ecográfico de la glándula, el PSA y los ratios del PSA (PSA libre, \% PSA libre/PSA total, PSA complex, densidad del PSA, velocidad del PSA).

Ocasionalmente un adenocarcinoma prostático es descubierto de modo fortuito y no esperado (incidental) al analizar la pieza quirúrgica enucleada tras la APS realizada por un supuesto diagnostico de patología benigna. La prevalencia del adenocarcinoma prostático incidental tras la APS es variable en la literatura, metodología empleada en el estudio, criterios de selección, distintos países, casuísticas, y otros parámetros, con una diferencia entre el 1-12\% (1-6). Es entonces cuando el cirujano se encuentra con dos problemas principales; el primero deriva de la información facilitada al paciente previamente a la intervención ("extirpación de un tumor benigno"), ya que en ninguno de los documentos de información y consentimiento quirúrgico que hemos podido revisar consta la posibilidad de detección de un adenocarcinoma prostático oculto y no sospechado; el segundo problema y más importante es la actitud a tomar en estos casos, ya que no existe un consenso claro sobre el tratamiento y manejo del cáncer de próstata incidental y además los factores pronósticos útiles varían de unos casos a otros (2). Solo parecen existir ciertas "pautas" de actuación en cuanto a que pacientes ofertarles un determinado tratamiento y a cuales otros. Existen diversas modalidades o pautas de tra- 
tamiento o manejo del cáncer prostático incidental, que abarcan desde el seguimiento (más o menos activo) sin tratamiento ("esperar y ver"), el tratamiento hormonal, la radioterapia externa o la cirugía radical (Prostatectomía Radical). Otras alternativas mas recientes como la braquiterapia, crioterapia y los HIFU no parecen, de momento, sin embargo recomendados en estos casos, aunque su corta experiencia no permite la toma de conclusiones definitivas.

En algunos de estos casos, el tratamiento con intención curativa, ya sea con cirugía o con radioterapia, supondrá un sobretratamiento (25-30\%) $(3,4)$ mientras que en otros la actitud de observación y seguimiento constituirá un riesgo de progresión (16 - 36\%) $(2,3,5$ y 6) difícil de predecir (3).

En este trabajo retrospectivo comparamos la prevalencia de Cáncer de Próstata Incidental (CPI) en dos grupos de pacientes sometidos a una APS por supuesta HBP sintomática. Un grupo son los pacientes en los que no se realizó una biopsia prostática por no estar esta indicada (tacto digito rectal normal y $\mathrm{PSA}<4 \mathrm{ng} / \mathrm{ml}$ ), y otro grupo en el que si se realizó Biopsia Prostática Ecodirigida (BPE) transrectal previa a la APS (tacto rectal patológico y/o PSA elevado $\geq 4 \mathrm{ng} / \mathrm{ml}$ ). Así mismo, también hemos analizado el tratamiento y la evolución posterior de dichos pacientes con $\mathrm{CPI}$.

\section{MATERIAL Y MÉTODOS}

Durante un periodo de 6 años consecutivos (1996 - 2001) se evaluaron retrospectivamente 549 pacientes intervenidos en nuestro servicio mediante una APS por una "supuesta" HBP sintomática no resuelta de modo totalmente satisfactorio con tratamiento farmacológico conservador. Edad media del grupo 71,2 años (rango 55 - 90).

Durante este periodo se intervinieron en nuestro servicio un total de 1593 pacientes de "supuesta" HBP sintomática. Se realizaron una media de 266 cirugías por año, con una distribución media de $92(34,5 \%)$ cirugías abiertas/año o APS y 174 $(65,5 \%)$ endoscópicas/año o resecciones-incisiones transuretrales de próstata (RTU-ITUP). Esta diferencia de los porcentajes entre cirugía endoscópica y cirugía abierta en nuestro hospital no es similar a las tendencias generales de otros centros en las fechas del estudio. Debido a que nuestro centro deriva, en algunas ocasiones y periodos determinados, la cirugía endoscópica en pacientes de "bajo riesgo quirúrgico" a hospitales adscritos y concertados, la diferencia se modifica sensiblemente. Dichos casos no intervenidos en nuestro centro no han formado parte de este estudio ni han constituido un sesgo de selección en el mismo (al tratarse únicamente de cirugía endoscópica y no abierta).

El volumen prostático se determinó por ecografía abdominal (realizada por un radiólogo) y por ecografía transrectal (realizada por urólogo) en los casos en que se realizó biopsia prostática transrectal ecodirigida (BPE) previa a la APS. Todos los pacientes tenian determinación previa de PSA.

De los 549 pacientes intervenidos por cirugía abierta (APS), un grupo precisó ser biopsiado previamente a la intervención como método de cribaje de patología prostática maligna, siendo el resultado de benignidad y realizándose posteriormente la cirugía. El resto de los pacientes no fueron biopsiados por no estar indicada dicha intervención de cribaje. Las biopsias fueron realizadas con control ecográfico transrectal tomando entre 6 y 10 cilindros prostáticos a lo largo de los 6 años del estudio retrospectivo. La indicación para realizar la biopsia fue un hallazgo anormal en el examen dígito rectal y/o un PSA alterado (PSA total $\geq 4 \mathrm{ng} / \mathrm{ml} ;<25 \%$ PSA total/libre). En principio un tacto rectal anormal, y así lo consideramos nosotros a pesar de que algunos autores sugieren que tiene escaso valor predictivo para detectar cáncer de próstata (7), es indicación para realizar una biopsia prostática independientemente del valor del PSA (8), ya que un porcentaje importante de pacientes (14 - 30\%) con un tacto rectal anormal y niveles de PSA entre $1-4 \mathrm{ng} / \mathrm{ml}$ tendrán cáncer de próstata en la biopsia (9).

Debido a que muchos pacientes con sintomatología prostática son biopsiados como parte del protocolo de despistaje de patología maligna prostática y no con la intención de una cirugía (pacientes asintomáticos), se consideró a la BPE como la biopsia "previa a la APS" cuando esta fue realizada como máximo un año antes de la cirugía abierta. Los pacientes que en algún momento de la evolución de su patología prostática habían sido biopsiados mas de un año antes de la cirugía abierta como método de despistaje tumoral prostático, fueron considerados como no biopsiados "previamente" a la APS para el estudio actual.

De los 549 pacientes a los que se les realizó una APS objeto del estudio, 291 (53\%) no precisaron la realización de una BPE como despistaje de patología maligna por no estar esta indicada bajo los criterios de un examen dígito rectal normal y un PSA $<4 \mathrm{ng} / \mathrm{ml}$. En algunos casos, y debido a la heterogenicidad de los urólogos responsables de los pacientes y de las consultas y revisiones médicas, pacientes con PSA $\geq 4 \mathrm{ng} / \mathrm{ml}$ no fueron biopsiados pre- 
viamente a la APS. Dicha decisión se tomó en base a la edad de los pacientes ( $\geq 75$ años en todos los casos), volumen prostático por ecografía abdominal (> de $60 \mathrm{~cm}^{3}$ ), tacto rectal (normal en todos los casos), y formas moleculares del PSA (PSA libre > 1, \% PSA libre/PSA total $>25 \%$ ). El resto de los pacientes, 258 $(47 \%)$, fueron previamente biopsiados como método de cribaje ante sospecha de patología tumoral maligna. Los criterios seguidos para la indicación de dicha biopsia fueron: tacto dígito rectal anormal o sospechoso de patología tumoral maligna en un $7,4 \%$ y $\mathrm{PSA} \geq 4 \mathrm{ng} / \mathrm{ml}$ o PSA "límite" (próximo a $4 \mathrm{ng} / \mathrm{ml}$ ) con alteración de alguna de sus formas moleculares (PSA libre < 1, \% PSA libre/PSA total $<25 \%$ ) en un $92,6 \%$

Fueron excluidos del estudio aquellos candidatos a APS en los que la biopsia prostática fue positiva para cáncer de próstata. También fueron excluidos los pacientes a los que se les detectó en la biopsia lesiones precancerosas (atipias, PIN anaplásico) debido al alto riesgo de desarrollo de cáncer de próstata (10 - 12). Solo fueron incluidos en el estudio pacientes a los que se les realizó APS con biopsia previa negativa para cáncer de próstata o lesiones premalignas, es decir, aquellos cuyas biopsias fueron de hiperplasia benigna y/o prostatitis aguda o crónica.

A las piezas enucleadas tras las cirugías (APS) se les realizó un estudio anatomopatológico completo (peso, medidas, estudio macroscópico y microscópico...etc.) con el fin de descartar la existencia de un tumor prostático incidental. El método seguido en nuestro centro para el estudio de las piezas quirúrgicas es el siguiente: las piezas son incluidas para el estudio macroscópico en su totalidad, realizándose cortes o secciones (step-sections) cada 3 - $5 \mathrm{~mm}$ (aproximadamente un corte cada 6-8 gramos de peso), analizando microscópicamente, si existen, las zonas sospechosas de patología tumoral, o un corte cada 8-10 gramos de tejido si no se evidencia ningún área sospechosa (y no menos de 7-10 cortes para estudio microscópico por pieza). En caso de hallazgo de tumor prostático incidental, las piezas son nuevamente estudiadas en totalidad (si el tamaño de la pieza lo permite), mas en profundidad y cuidadosamente con cortes o secciones cada 1 - $2 \mathrm{~mm}$ para un nuevo estudio microscópico y para confirmar los hallazgos y asegurar el estadio.

Los resultados obtenidos fueron tratados mediante estudio estadístico empleando el test de Chi cuadrado.

\section{RESULTADOS}

El volumen prostático medio determinado por ecografía abdominal fue de $110,3 \mathrm{~cm} 3(112,8$ $\mathrm{cm} 3$ en el grupo de los biopsiados y $108,1 \mathrm{~cm}^{3}$ en

\section{TABLA I. DISTRIBUCIÓN POR GRUPOS DE LAS MEDIAS DE DIVERSOS PARÁMETROS DE LOS PACIENTES DEL ESTUDIO. CPI = CÁNCER PRÓSTATA INCIDENTAL}

\begin{tabular}{|l|c|c|c|c|c|}
\hline Grupos. & $\mathbf{n}$ & Edad media. & $\begin{array}{c}\text { Ecografía } \\
\text { Abd. }\left(\mathrm{cm}^{3}\right)\end{array}$ & $\begin{array}{c}\text { Pieza } \\
\mathrm{Qx}(\mathrm{gs})\end{array}$ & $\begin{array}{c}\text { PSA } \\
\mathrm{ng} / \mathrm{ml}\end{array}$ \\
\hline Total. & 549 & 71.2 & 110.3 & 75.6 & $\begin{array}{c}12.8 \\
(0.7-129)\end{array}$ \\
\hline No biopsiados. & $\begin{array}{c}291 \\
(53 \%)\end{array}$ & 71.5 & 112.2 & 73.6 & $\begin{array}{c}4.8 \\
(0.7-6.8)\end{array}$ \\
\hline Biopsiados. & $\begin{array}{c}258 \\
(47 \%)\end{array}$ & 70.9 & 108.3 & 77.9 & $\begin{array}{c}22.9 \\
(4.2-129)\end{array}$ \\
\hline No CPI. & $\begin{array}{c}524 \\
(95,4 \%)\end{array}$ & 71.1 & 111.5 & 76.2 & $\begin{array}{c}13.1 \\
(0.7-129)\end{array}$ \\
\hline CPI. & $\begin{array}{c}25 \\
(4,5 \%)\end{array}$ & 75.2 & 85.5 & 64.9 & $\begin{array}{c}7.7 \\
(2.8-30)\end{array}$ \\
\hline CPI No biopsiados. & $\begin{array}{c}19 \\
(3,4 \%)\end{array}$ & 75.1 & 81.5 & 55.4 & $\begin{array}{c}4.2 \\
(2.8-6.8)\end{array}$ \\
\hline CPI biopsiados. & 6 & 74.5 & 98.3 & 95.2 & $\begin{array}{c}18.8 \\
(7.9-30)\end{array}$ \\
\hline
\end{tabular}


el grupo de los no biopsiados), el determinado por ecografía transrectal en los pacientes a los que se les realizó BPE transrectal fue de $92,8 \mathrm{~cm}^{3}$, y el de las piezas quirúrgicas enucleadas de 75,6 gramos de media.

La prevalencia global de CPI en nuestra serie en el periodo del estudio de seis años fue de 25 pacientes de entre las 549 APS analizadas $(4,55 \%)$, con una incidencia anual de 4,1 casos/año. Edad media de los pacientes con CPI 75,2 años (63 - 90). El volumen prostático enucleado medio de los pacientes con CPI fue 64,9 gramos (32 - 159). (Tabla I)

Tras el hallazgo de CPI en un primer estudio anatomopatológico, las piezas fueron nuevamente evaluadas microscópicamente en profundidad para la confirmación del diagnóstico y asegurar el estadio. Tras esta nueva revisión cuidadosa, ningún estadio tumoral fue reasignado en otro de grado mayor, lo que muestra el alto grado de fiabilidad del primer examen siempre y cuando se incluya para el estudio anatomopatológico la pieza enucleada en su totalidad. (Figura 1)

Por grupos, de los 25 pacientes con CPI, 19 $(76 \%)$ pertenecen al grupo de los no biopsiados previamente a la APS por no estar esta intervención indicada, lo que hace una prevalencia en este grupo del $3,46 \%$. El resto de los $\mathrm{CPI}, 6$ pacientes $(24 \%)$, pertenecen al grupo de los biopsiados, con una prevalencia en este grupo del 1,09\%. El motivo de la BPE en

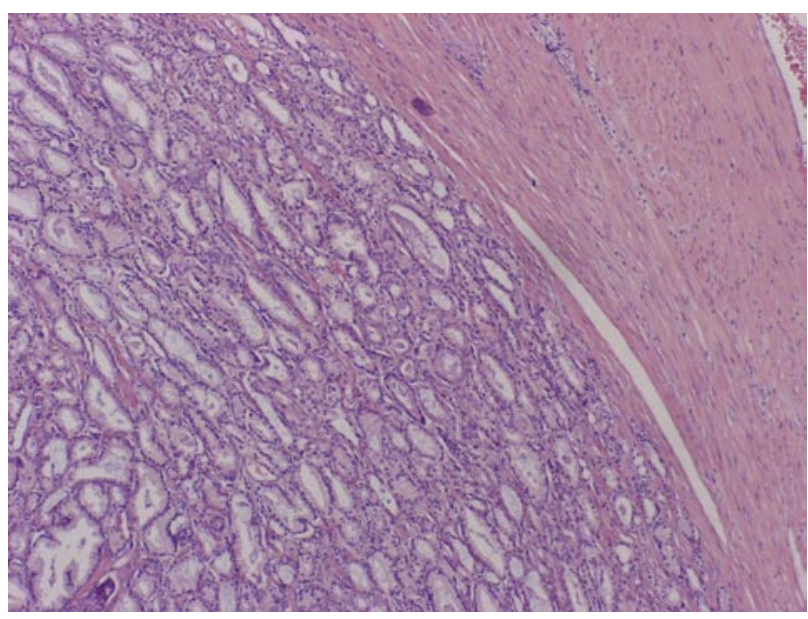

FIGURA 1. Foco microscópico (40x) de adenocarcinoma de próstata incidental en una pieza de adenomectomía suprapúbica. Tumor muy bien delimitado, mostrando glándulas de pequeño tamaño, de disposición irregular y próximas entre si, grado de Gleason 3+2 (5). estos pacientes (sextante en cinco casos y ampliada a 10 cilindros en un caso) fue PSA elevado en todos con una media de $18,8 \mathrm{ng} / \mathrm{ml}(7,9-30)$ con un volumen prostático enucleado medio de 95,2 gramos $(70$ - 159). El PSA medio de los pacientes no biopsiados con CPI posteriormente fue de $4,2 \mathrm{ng} / \mathrm{ml}(2,8-6,8)$ con un volumen prostático enucleado medio de 55,4 gramos (30 - 95). (Tabla I)

La diferencia de tiempo entre la realización de la BPE y la cirugía abierta fue de 114,8 días (6246). Como ya indicamos, consideramos válida a la BPE para este estudio (biopsia "previa" a la APS) cuando esta diferencia es inferior a un año. Por ello, dos pacientes con CPI fueron considerados como no biopsiados previamente a la APS al haberse detectado en el curso de la evolución de su patología prostática una biopsia, con una diferencia de tiempo entre esta y la cirugía abierta de mas de dos y tres años.

Por edades, la prevalencia máxima (28\%) se dio entre el grupo de los pacientes comprendidos entre los 66-70 años (media de 67,5), seguido (24\%) por los grupos comprendidos entre los $71-75$ años (media 73,8$)$ y $(20 \%)$ 76-80 (media 77,6$)$.

Por estadios, 22 casos correspondieron a un estadio pTla $(88 \%)$ con volúmenes tumorales detectados inferiores al $5 \%$ del volumen enucleado. El resto, 3 casos, correspondieron a un estadio $\mathrm{pT} 1 \mathrm{~b}$ $(12 \%)$, con volúmenes tumorales en los tres casos del $10 \%$ aproximadamente en las piezas enucleadas. Estos tres casos de pacientes en estadio pTl b corresponden a pacientes del grupo de los no biopsiados previamente a la APS. Todos los pacientes biopsiados previamente con CPI posterior en las piezas enucleadas se encontraban en estadio pTla.

El Gleason medio fue de 4,5, con una moda de 4 y un rango entre 3 y 7 : Gleason $\leq 4=13$ casos $(52 \%)$; Gleason 5-6 = 15 casos (44\%); Gleason 7 $=1$ caso $(4 \%)$. El score de Gleason medio de los pacientes no biopsiados previamente a la APS fue de 4,6 y en el grupo de los biopsiados de 4,1. El score medio de Gleason de los casos con estadios pTla fue de 4,3 y de los pT1b 5,6. (Figura 2).

Por tamaños de las piezas quirúrgicas enucleadas, la distribución de los CPI fue homogénea (13), existiendo prácticamente el mismo número de casos en las piezas de menos de 60 gramos $112 \mathrm{ca}$ sos) que en las piezas de más de 60 gramos (13 casos). El volumen medio de los adenomas enucleados en los que se detectó CPI fue de 64,9 gramos, diferencia inferior al volumen medio de las APS totales del estudio $(75,6$ gramos). 
Respecto al tratamiento de los pacientes con $\mathrm{CPI}, 21$ pacientes no se trataron (84\%) y se vigilaron estrechamente con el fin de detectar precozmente una posible recidiva o progresión tumoral ("esperar y ver"). Dos pacientes (8\%) fueron tratados desde el diagnostico de CPI con Bloqueo Androgénico Completo (antiandrogeno+agonista LHRH) al tener volúmenes tumorales incidentales mayores del $5 \%$ (estadios pT 1b). Los otros dos pacientes restantes $(8 \%)$ fueron tratados con finasteride desde el diagnostico de $\mathrm{CPI}$, ambos en estadio pTla. A ningún paciente de nuestra serie de adenomectomías (APS) se le realizó prostatectomía radical tras el diagnostico de CPI en las piezas enucleadas.

De los 25 pacientes con CPI, dos pacientes se perdieron para el seguimiento tras 24 y 30 meses sin progresión $(8 \%)$ y se observaron en el curso de la evolución de los casos 5 muertes no relacionadas con patología urológica (20\%).

Tras una media de seguimiento de 48, 1 meses (22 - 96 meses), detectamos 3 progresiones tumorales $(12 \%)$. Los tres pacientes que progresaron pertenecen al grupo de los de vigilancia sin tratamiento (21 pacientes), lo que representa una prevalencia de progresión en este grupo ("esperar y ver") del $14,2 \%$, y una prevalencia global (25 pacientes $=4$ tratados y 21 no tratados) del $12 \%$. El tiempo medio hasta la progresión fue de 40,6 meses (12 - 72 meses). Ningún paciente al que se le realizó previamente a la APS una BPE progresó. Los 3 casos en los que se detectó progresión tumoral durante el seguimiento pertenecen a pacientes diagnosticados de $\mathrm{CPI}$ en estadios pTl a. Ningún paciente en estadio pTlb al diagnostico progresó.

Respecto a la progresión tumoral, esta se detectó a los 12, 38 y 72 meses del seguimiento respectivo (media 40,6 meses). En los tres casos se confirmó la progresión tumoral mediante BPE transrectal indicada por PSA elevado (media $8 \mathrm{ng} / \mathrm{ml}$, rango $5,7-12,1)$ y además en uno de ellos por un examen dígito rectal "sospechoso" (aunque insistimos en el dudoso valor y difícil interpretación del tacto rectal en pacientes con una APS previa). La elevación media del PSA en los tres casos fue de $\times 1,64 \mathrm{ng} / \mathrm{ml}$ (rango $\times 1,5-x 1,79$ ) respecto al PSA inicial previo a la APS, valor que no indicó la necesidad de realizar BPE y por ello pertenecen los tres pacientes, como ya se ha indicado, al grupo de los no biopsiados previamente a la APS. El score de Gleason se elevó en las progresiones tumorales una media de 3,6 puntos (rango 3-4 puntos) con respecto al Gleason de la pieza enucleada de la APS en donde se detectó el CPI (Gleason medio del $\mathrm{CPI}=3,6$; Gleason medio de la progresión detectada mediante $\mathrm{BPE}=7,3)$.

En total, durante el seguimiento de los pacientes tras el diagnóstico de CPI, en 4 de los 25 (16\%) se realizó biopsia prostática ecodirigida (BPE) para descartar progresión tumoral. En todos los casos (4) el motivo de la indicación de la BPE fue un aumento del PSA, dudando como se ha indicado, del valor del
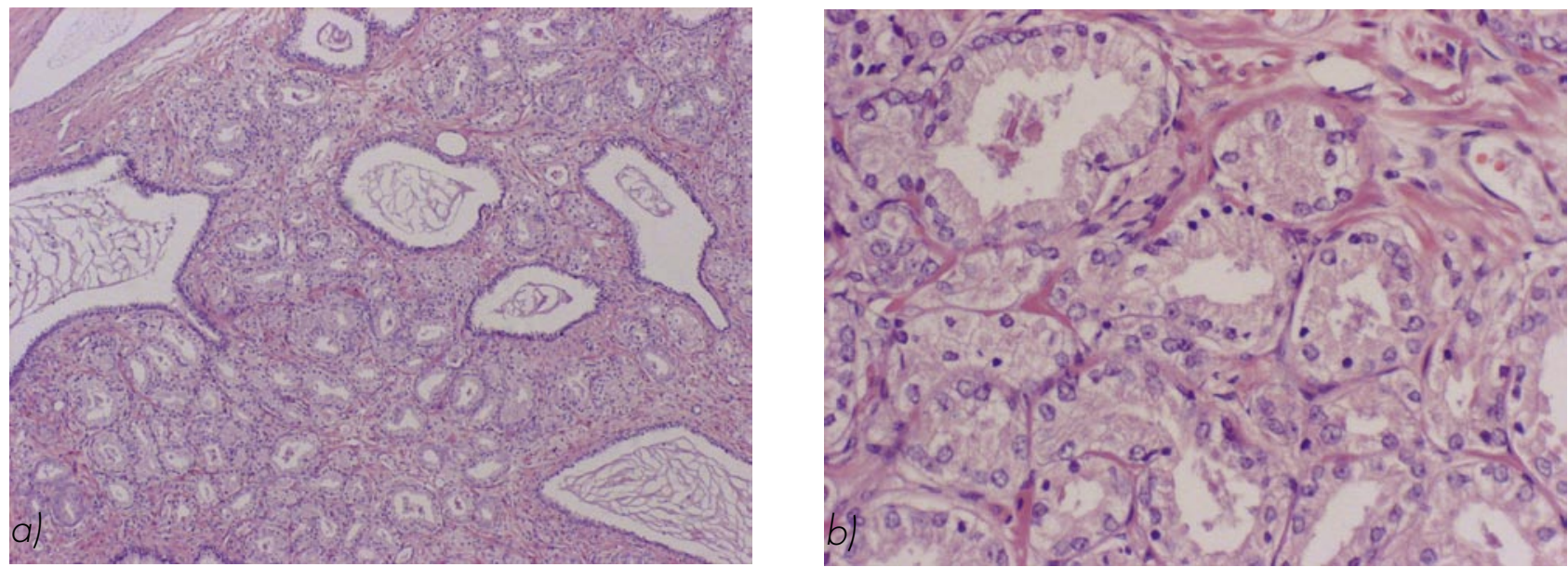

FIGURA 2. 2a): Foco microscópico (40x) de adenocarcinoma de próstata incidental en una pieza de adenomectomía suprapúbica. Se observa la coexistencia de glándulas tumorales (pequeñas e irregulares

que se sitúan próximas entre sil, y glándulas hiperplasicas (grandes y dilatadas).

2b): Mismo foco a mayor aumento (200x). Se evidencian glándulas pequeñas e irregulares, situadas muy próximas entre si (sin a penas estroma) y nucleolo muy evidente, grado de Gleason 3+3 (6). 
tacto rectal en estos casos (elevación media del PSA de $\times 1,78 \mathrm{ng} / \mathrm{ml}$ respecto al PSA previo a la APS que en ninguno de los casos indicó la necesidad de BPE previa a la APS). En 2 de estos 4 casos, una primera BPE tras el diagnóstico de CPI fue negativa (realizada por elevación media del PSA de $\times 1,77 \mathrm{ng} / \mathrm{ml}$ respecto al PSA previo a la APS), y una segunda BPE posterior realizada por persistencia e incremento de los criterios de sospecha de progresión tumoral confirmó la presencia de tumor (realizada por elevación media del PSA de $\times 3,57 \mathrm{ng} / \mathrm{ml}$ respecto al PSA previo a la APS). Dichas rebiopsias se realizaron a los 38 y 72 meses de seguimiento respectivamente. En los otros dos casos, en uno de ellos la BPE fue negativa, normalizándose posteriormente el PSA y manteniéndose posteriormente sin criterios de sospecha de progresión. En el otro caso, una única BPE confirmó la presencia de tumor prostático. (Figura 3).

En lo que hace referencia al tratamiento de las progresiones, el paciente de menor edad 166 años) fue candidato a linfadenectomía iliaco-obturatriz bilateral de estadiaje tumoral con bloqueo hormonal máximo previo con resultado anatomopatológico negativo (sin evidencia de enfermedad tumoral ganglionar), realizándose posteriormente Radioterapia externa complementaria (70 Gy). El seguimiento tras este tratamiento es de 48 meses sin tratamiento, sin evidencia de nueva progresión y con controles periódicos normales. A los otros dos pacientes se les trató

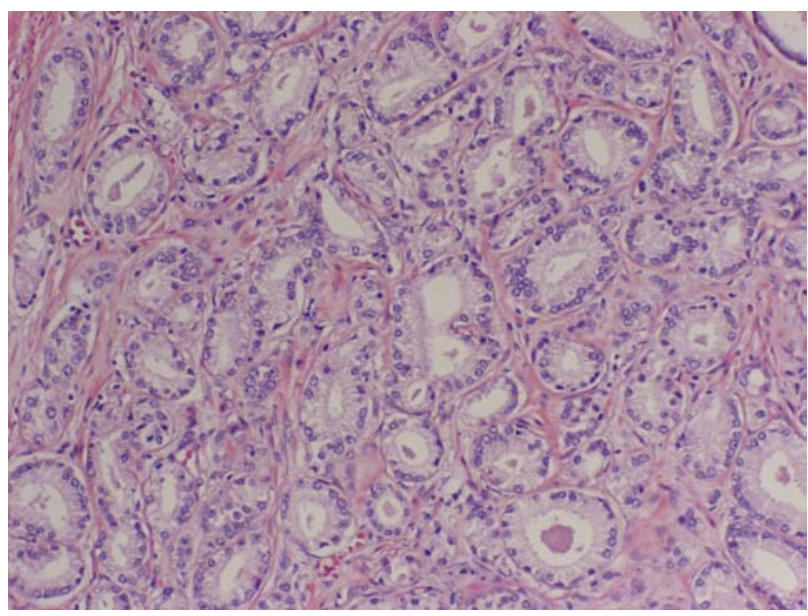

FIGURA 3. Adenocarcinoma en una biopsia de próstata realizada durante el seguimiento de un paciente con tumor de próstata incidental (100x).

Se evidencian glándulas pequeñas con una única capa de células epiteliales y que se sitúan muy próximas, de forma irregular y que en ocasiones se fusionan, grado de Gleason 3+4 (7). desde el diagnostico de la progresión tumoral con bloqueo hormonal máximo durante 12 meses, pasando uno de ellos a una pauta de tratamiento hormonal intermitente durante otros 12 meses mas al controlar la progresión de la enfermedad durante los primeros 12 meses de tratamiento. En el otro paciente en que se empleó el bloqueo hormonal máximo se realizó, tras 12 meses de tratamiento con mala tolerancia debida a los efectos secundarios y sintomatología obstructiva, una resección transuretral desobstructiva (RTU-P) junto con orquiectomía subalbugínea bilateral. El estudio anatomopatológico de los fragmentos de la RTU-P mostró varios de ellos infiltrados por un adenocarcinoma Gleason $9(4+5)$. El seguimiento actual es de 24 meses más tras la cirugía, sin tratamiento en la actualidad y sin evidencia de nueva progresión tumoral.

Tras el tratamiento de las progresiones de la enfermedad, ninguno de los tres pacientes ha continuado progresando, estando la enfermedad en la actualidad controlada en los tres con una media de seguimiento desde la detección de la progresión del $\mathrm{CPI}$ de 32 meses (24 - 48 meses).

Con un periodo medio de seguimiento de 48,1 meses (22 - 96 meses) no se detectó ningún caso de mortalidad cáncer de próstata específica (con dos pacientes perdidos para el seguimiento tras 24 y 30 meses sin progresión). (Tabla II)

Durante dicho periodo de seguimiento, en 6 de los 25 casos (24\%), se detectó en los pacientes otras neoplasias diferentes a la prostática: carcinoma epidermoide retroauricular; adenocarcinoma de recto; linfoma no Hodgkin; adenocarcinoma de páncreas; y dos casos de carcinomas de células transicionales infiltrantes de vejiga. Estos últimos casos fueron sometidos a cistoprostatectomía radical, con buena evolución posterior en un caso y progresando la enfermedad vesical hasta el exitus en el otro (exitus no relacionado con el CPI). El análisis anatomopatológico de las piezas quirúrgicas (cistoprostatectomía) no evidenció en ambos casos la persistencia de adenocarcinoma prostático tras su revisión cuidadosa.

\section{DISCUSIÓN}

El manejo correcto y protocolizado de los pacientes con CPI es actualmente un problema no resuelto, aunque parece existir cierto consenso en la literatura respecto a ofrecer tratamiento quirúrgico a los pacientes mas jóvenes, con buena expectativa de vida (por encima de los 10-15 años) y en casos de tumores pobremente diferenciados o agresivos $(2,3$, $13,15-18)$. 


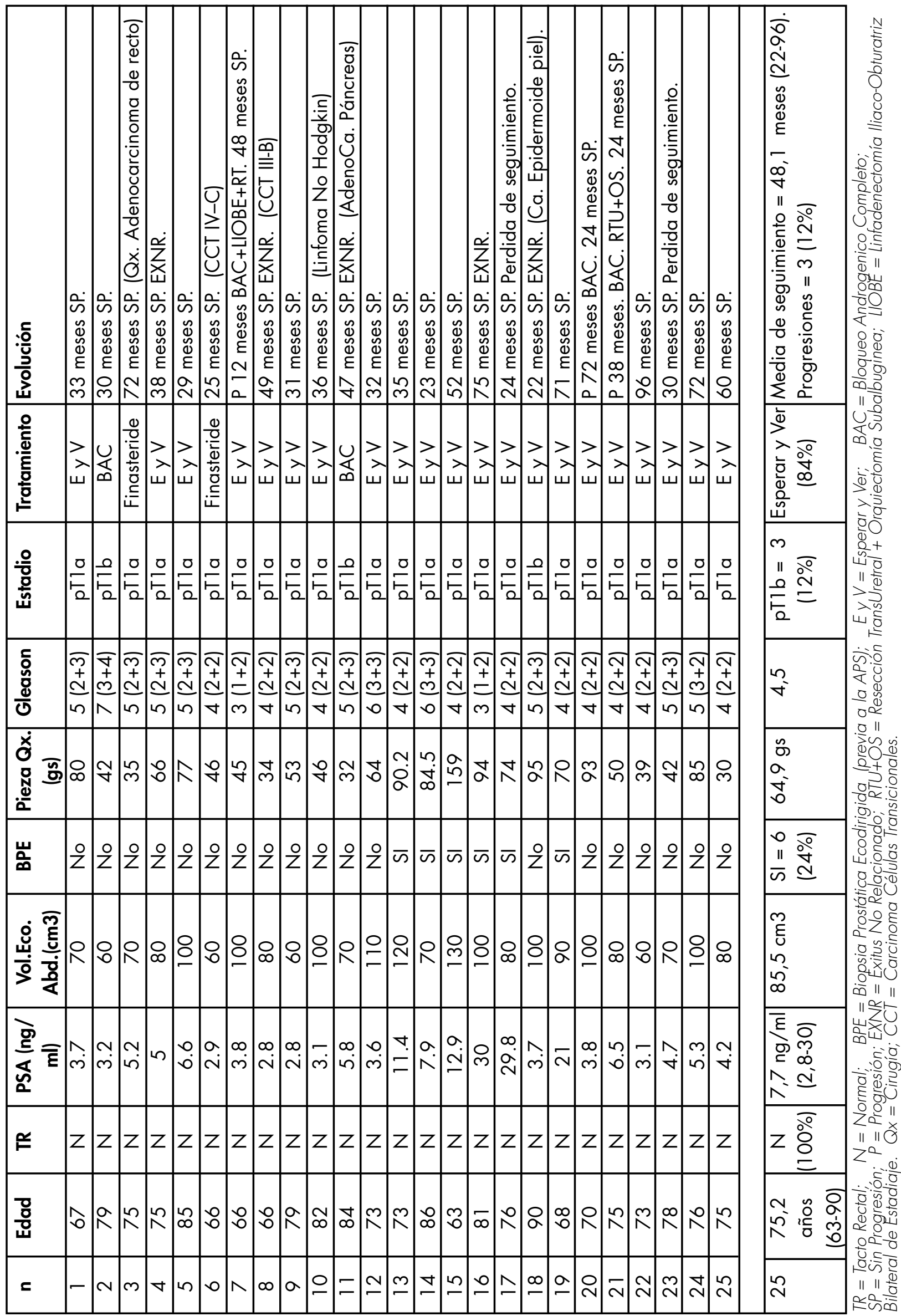


Nos parece lógico incidir en la revisión anatomopatológica cuidadosa de los especímenes quirúrgicos enucleados con $\mathrm{CPI}$ para confirmar el diagnostico y asegurar el estadio. Para ello es fundamental, a parte de la experiencia del patólogo y de la sospecha del posible hallazgo de un CPI, un correcto protocolo de estudio junto con la inclusión de la pieza en su totalidad con revisión posterior ampliada y cuidadosa en caso de hallazgo de CPI.

Algunos autores intentan justificar la realización de BPE previa a la APS por encontrar una prevalencia de cáncer de próstata en estos pacientes candidatos a cirugía y PSA elevado superior al 10 $\%$ (19). Esta corriente trata de reducir al máximo la prevalencia posterior de CPI, aumentando con ello el porcentaje de pacientes diagnosticados en estadio pTlc y susceptibles de algunos de los tratamientos curativos actualmente aceptados.

El concepto de punto de corte del PSA en 4 $\mathrm{ng} / \mathrm{ml}$ esta actualmente considerado como anticuado. Un porcentaje importante de pacientes (superior al $20 \%$ ) son actualmente diagnosticados de cáncer de próstata con valores de PSA inferiores a $4 \mathrm{ng} / \mathrm{ml}$. Por ello el punto de corte se empieza a establecer en niveles más inferiores y próximos a $2,5 \mathrm{ng} / \mathrm{ml}$. Por otro lado, pacientes con grandes adenomas prostáticos presentan valores de PSA elevados o "borderline". Nosotros aumentamos la sensibilidad y especificidad de la BPE usando las formas moleculares del PSA, la fracción libre del PSA $(<1 \mathrm{ng} / \mathrm{ml}$ ) y el cociente PSA libre / PSA total $(<25 \%)$, para tomar la decisión de realizar o no la biopsia. El porcentaje de PSA libre es efectivo para reducir el número de biopsias prostáticas innecesarias, aunque depende en gran medida del punto de corte que se tome como referencia. Existe un "factor de conversión" por unidad de volumen prostático propuesto por Collins y cols. (20) (upper limit PSA $=$ unidad de volumen prostático en $\mathrm{cm}^{3} \mathrm{X} 0,072$ ), para ajustar los niveles de PSA al volumen de la próstata de cada paciente. Por lo general nosotros no empleamos dicho método para la toma de decisiones.

Probablemente parte de esta problemática sobre el tratamiento o no de los CPI se resuelva en un futuro con la disminución de los valores de PSA recomendados para la valoración de biopsia, con la identificación y estudio de los factores pronósticos y los factores de progresión, pudiendo entonces identificar a los pacientes de alto riesgo de progresión tumoral que se beneficien de un determinado tratamiento frente a los de bajo riesgo en los que prevalezca la opción de seguimiento vigilado. Aún así, el descenso del rango de PSA y su uso indiscriminado en el screening prostático creará una controversia al incrementar el sobrediagnóstico en pacientes en los cuales su cáncer prostático nunca sería "clínicamente" diagnosticado (21).

Como era de esperar, la prevalencia de CPI en nuestro estudio en el grupo de los pacientes no sometidos a BPE previa a la APS fue superior a la prevalencia del grupo de los biopsiados $13,46 \%$ frente a $1,09 \%$ ). Dicha diferencia se aproximó, debido al escaso número de casos de CPI del estudio, a la significación estadística tras someterse al test $\mathrm{Chi}$ cuadrado con una $p=0,0803$. La prevalencia de CPI en pacientes intervenidos de APS en los diversos estudios revisados oscila entre el $1,1 \%$ y el $12 \%$ (14-18, 22-24). La prevalencia en nuestra serie es similar a la de otros estudios con casuísticas y metodologías parecidas, con la excepción de que en ninguno de los estudios revisados se hace referencia a dos grupos de pacientes diferentes según se hayan sometido a BPE previa a la APS o no.

Por tamaños de las piezas quirúrgicas enucleadas, no encontramos nosotros las diferencias que algunos autores sugieren, con un aumento significativo de casos de CPI en glándulas de 30 - 40 gramos (13). La distribución de los CPI en nuestra serie fue homogénea, existiendo prácticamente el mismo número de casos en las piezas de menos de 60 gramos (12 casos) que en las piezas de más de 60 gramos (13 casos).

El probable motivo del fallo en la detección del cáncer de próstata en los pacientes biopsiados y con posterior CPI (falsos negativos) fue la escasa carga tumoral, alojada en la zona transicional prostática, de los CPI detectados en las piezas enucleadas (todos estadios pTla con volúmenes tumorales inferiores al $5 \%$ ) junto al hecho de tratarse de próstatas de grandes volúmenes y por ello candidatas a cirugía abierta (volumen enucleado medio de 95,2 gramos). Además, el $20 \%$ aproximado de los cánceres de próstata se originan en la zona transicional de la glándula, siendo infrecuente su detección por biopsia debido al aumento importante de esta zona en pacientes candidatos a una APS. Otro factor que influyó fue la técnica empleada para la biopsia, ya que el sextante descrito por Hodge et al 25,26 tiene una tasa de falsos negativos próxima al $30 \%$. Existe relación inversa entre el tamaño de la próstata y la probabilidad de detectar cáncer de próstata (27-29). El tamaño de la glándula afecta al rendimiento de la biopsia, con mayor tasa de falsos negativos en las glándulas de tamaños más grandes (30).

Este trabajo muestra las diferencias existentes, aproximándose a la significación estadística, entre ambos grupos, los pacientes biopsiados previamente y los no biopsiados, no encontrando artícu- 
los iguales en la literatura, por lo que no podemos comparar los resultados con los de otros grupos. Esta importante diferencia podría justificar la realización de BPE previas a la APS en pacientes con PSA elevado, sospechoso o límite, o ante la duda en el tacto rectal. Todo ello surge en un intento de diagnosticar precozmente a pacientes con cánceres de próstata subclínico y organoconfinados, reduciendo al máximo la prevalencia de CPI. La BPE previa a la APS redujo en nuestra serie en mas de un $68 \%$ (del $3,46 \%$ al 1,09\%) la prevalencia de CPI posterior, aumentando con ello el diagnostico precoz de pacientes en estadio pTlc candidatos a alguno de los tratamientos curativos. En nuestro caso, la mejor "prevención" de CPI fue el cribado mediante biopsia de los pacientes sospechosos (31). Así mismo, los pacientes biopsiados con CPI posterior mostraron scores medios de Gleason inferiores respecto al grupo no biopsiado $(4,16$ frente a 4,68$)$, además de que en ningún paciente de los biopsiados el volumen tumoral en la pieza de la APS fue mayor del $5 \%$, es decir, ninguno mostró un estadio tumoral superior a un pTla.

El score de Gleason se elevó de manera importante en las progresiones tumorales (media de 3,6 puntos) con respecto al Gleason de la pieza enucleada de la APS en donde se detectó el CPI (Gleason medio del $\mathrm{CPI}=3,6$ con rangos de 3-4; Gleason medio de la progresión detectada mediante BPE = 7,3 con rangos de 7-8). Con ello se evidencia que, probablemente, la progresión de la enfermedad no fue por recidiva (nuevo tumor) del cáncer prostático detectado incidentalmente en la pieza de la APS, sino una verdadera progresión del tumor primario incidental residual, no extraído completamente en la pieza de la APS.

La progresión de la enfermedad en pacientes no tratados (grupo "esperar y ver") oscila en la literatura revisada entre el $16-36 \%(2,4,5)$, planteándose entonces diversas alternativas para el tratamiento. En nuestro estudio, la progresión fue del $14,2 \%$ en este grupo de pacientes no tratados tras el diagnostico de CPI (progresaron 3 de 21 pacientes), con una evolución actualmente favorable tras el tratamiento de rescate. Así pues, la progresión total 3 de los 25 pacientes del total de pacientes tratados y no tratados) de los CPI estadios pTla y pTlb es baja en nuestra serie $(12 \%)$, con una supervivencia cáncer de próstata específica del $100 \%$ tras una media de seguimiento de 48,1 meses (22 - 96 meses).

Algunos autores han recomendado un protocolo de seguimiento vigilado "activo", incluso con rebiopsias prostáticas en periodos de tiempo determinados o en caso de alteración de alguno de los parámetros de seguimiento (PSA, tacto rectal), benefi- ciándose los pacientes de un control individualizado basado en el riesgo de progresión de la enfermedad con el tiempo (32). Esta puede ser una actitud válida y recomendable muy especialmente en los pacientes con expectativas de vida mayores (sobretodo en pacientes con expectativas de vida por encima de los 15 años). La biopsia reglada post-cirugía en caso de CPI puede ser una indicación en estos pacientes, dado que el PSA y tacto rectal no son totalmente óptimos en ese periodo (tras la APS), y por otro lado se puede calibrar la posibilidad de tumores en la periferia o cápsula prostática.

Por último, consideramos necesario la modificación al respecto de los documentos de consentimiento quirúrgico informado con el fin de ampliar la información disponible a los pacientes, indicando en ellos la posibilidad de detección incidental de un cáncer de próstata y la posibilidad de precisar tratamientos posteriores complementarios o seguimientos a largo plazo.

\section{CONCLUSIONES}

La prevalencia global de CPI en nuestra serie durante los seis años del estudio fue de 4,55\% (25 pacientes de entre 549 APS). La prevalencia de CPI tras biopsia y APS por "supuesta" HBP sintomática fue de $1,09 \%$ frente al 3,46\% del mismo grupo de pacientes sometidos a una APS sin biopsia prostática previa. Dicha diferencia, un $68 \%$ menor $(p=0,0803$ en el test Chi cuadrado), apoya la realización, en pacientes seleccionados, de biopsia prostática previa a la APS para disminuir el riesgo de tumores.

Los CPI detectados en las piezas quirúrgicas fueron mínimos focos (microscópicos) muy bien diferenciados. Ningún paciente biopsiado previamente a la APS presentó un estadio tumoral superior al pTla. La BPE (10 cilindros) es un buen método de cribaje de cáncer de próstata. Mínimos tumores prostáticos microscópicos pueden no ser detectados en la BPE sobre todo cuando se trata de próstatas de gran volumen candidatas a cirugía abierta.

La progresión tumoral detectada en todos los casos mediante biopsia indicada por PSA elevado fue del $12 \%$, con buen resultado tras el tratamiento de rescate y sin evidencia de enfermedad tras una media de seguimiento de 32 meses (24 - 48 meses). El pronostico de estos CPI a corto-medio plazo es bueno precisando un seguimiento estrecho con TR (de difícil interpretación tras cirugía prostática) y sobretodo con PSA (32) y biopsias de seguimiento en pacientes con expectativa de vida mayor ("seguimiento vigilado activo"). 
La supervivencia global cáncer de próstata específica de los CPI en nuestra serie revisada fue del $100 \%$ con una media de seguimiento de 48,1 meses (22 - 96 meses).

\section{BIBLIOGRAFÍA y LECTURAS RECOMENDADAS ( ${ }^{*}$ lectura de interés $y^{* *}$ lectura fundamental)}

1. ROSS, N.R.; WENNEBERG, J.E.; MALENKA, D.J. y cols.: "Mortality and reoperation after open and transurethral resection of the prostate for BPH”. N. Engl. J. Med., 320: 1120, 1985.

*2. LEISINGER, H.J.: "Prostatic cancer stage T1. "Incidental carcinoma". Review of the literature and critical reappraisal of the classification system". Ann. Urol., 28: 229, 1994.

*3. WÜRNSCHIMMEL, E.; LIPSKY, H.: "Incidental carcinoma of the prostate: "wait and see" or radical prostatectomy?". Urologe, 31: 48, 1992.

4. NUÑEZ LÓPEZ, A.V.; OJEA CALVO, A.; DOMÍNGUEZ FREIRE, F. y cols.: "Clinical and biological progresion of incidental prostatic cancer (stage T1a)". Actas Urol. Esp., 27: 345, 2003.

5. MANSECK, A.; WIRTH, M.; THEISS, M. y cols.: "Radical prostatectomy in patients with incidental prostate carcinoma". Urol. Int., 53: 196, 1994.

*6. ROY, C.R.; HORNE, D.; RAIFE, M. y cols.: "Incidental carcinoma of prostate, Long-term followup". Urology, 36: 210, 1990.

7. SCHROEDER, F.H.; VAN DER MAAS, P.; BEEMSTERBOER, P. y cols.: "Evaluation of the digital rectal examination as a screening test for prostate cancer". J. Natl. Cancer Inst., 90: 1817, 1998.

8. RICHIE, J.P.; CATALONA, W.J.; AHMANN, F.R. y cols.: "Effect of patient age on early detection of prostate cancer with serum prostatespecific antigen and digital rectal examination". Urology, 42: 365, 1993.

9. SCHROEDER, F.H.; VAN DER CRUIJSENKOETER, I.; DE KONING, H.J. y cols.: "Prostate cancer detection at low prostate specific antigen". J. Urol., 163: 806, 2000.

10. CHAN, T.Y.; EPSTEIN, J.I.: "Follow-up of atypical prostate needle biopsies suspicious for cancer". Urology, 53: 351, 1999.

11. PARK, S.; SHINOHARA, K.; GROSSFELD, G.D. y cols.: "Prostate cancer detection in men with prior high grade prostatic intraepithelial neoplasia or atypical prostate biopsy”. J. Urol., 165: 1409, 2001.

12. ICZKOWSKI, K.A.; BOSTWICK, D.G.: "Prostate biopsy 1999: strategies and significance of pathological findings". Semin. Urol. Oncol., 17: 177, 1999.

*13. STEINBERG, G.D.; BALES, G.T.; BRENDLER, C.B.: "Analysis of watchful waiting for clinically localized prostate cancer”. J. Urol., 159: 1431, 1998.

14. ORNSTEIN, D.K.; RAO, G.S.; SMITH, D.S. y cols.: "The impact of systematic prostate biopsy on prostate cancer incidence in men with symptomatic benign prostatic hyperplasia undergoing transurethral resection of the prostate". J. Urol., 157: 880, 1997.

*15. CHANG, C.P.; CHANG, Y.H.; CHIANG-HUNG, y cols.: "Incidental adenocarcinoma of the prostate: a retrospective analysis". Eur. Urol., 20: 282, 1991.

16. SCHURHOLZ, T.; SCHULZE, H.; FINKE, W. y cols.: "Clinical relevance of incidental prostate carcinoma. A retrospective study". Urologe A., 35: 315, 1996.

**17. 1STILLWELL, T.J.; MALEK, R.S.; ENGEN, D.E. y cols.: "Incidental adenocarcinoma after open prostatic adenectomy”. J. Urol., 141: 76, 1989.

**18. VAN ANDEL, G.; VLEEMING, R.; KURTH, K. y cols.: "Incidental carcinoma of the prostate". Semin. Surg. Oncol., 11: 36, 1995.

**19. FOWLER, J.E. Jr.; BIGLER, S.A.; KOLSKI, J.M.: "Prostate cancer detection in candidates for open prostatectomy". J. Urol., 160: 2107, 1998.

20. COLLINS, G.N.; LEE, R.J.; McKELVIE, G.B. y cols.: "Relationship between prostate specific antigen, prostate volumen and age in benign prostate". Br. J. Urol., 71: 445, 1993.

21. YAO, S.L.; LU-YAO, G.: "Understanding and appreciating overdiagnosis in the PSA era". J. Natl. Cancer Inst., 94: 958, 2002.

*22. VECCHIOLI SCALDAZZA, C.: "Incidental carcinoma of prostate. Study of 683 patients operated upon for benign prostatic hypertrophy”. Minerva Urol. Nefrol., 44: 109, 1992.

23. YONEDA, F.; MIYAKE, N.; TSUJIMURA, H. y cols.: "Clinical studies on cases of suprapubic prostatectomy". Hinyokika Kiyo, 33: 65, 1987.

24. MURANAKA, K.; TAKAEA, A.; OKANO, M. y cols.: "Clinical and statistical study on recent cases of suprapubic prostatectomy". Hinyokika Kiyo, 31: 969, 1985.

25. HODGE, K.K.; McNEAL, J.E.; TERRIS, M.K. y cols.: "Random systematic versus directed ultrasound guided transrectal core biopsies of the prostate". J. Urol., 142: 71, 1989.

26. LEVINE, M.A.; ITTMAN, M.; MELAMED, J. y cols.: "Two consecutive sets of transrectal ultrasound guided sextant biopsies of the prostate for the detection of prostate cancer". J. Urol., 159: 471, 1998. 
27. UZZO, R.G.; WEI, J.T.; WALDBAUM, R.S. y cols.: "The influence of prostate size on cancer detection". Urology, 46: 831, 1995.

28. KARAKIEWICZ, P.I.; BAZINET, M.; APRIKIAN, A.G. y cols.: "Outcome of sextant biopsy according to gland volume". Urology, 49: 55, 1997.

29. VALSHI, A.R.; WOJNO, K.J.; GILLESPIE, B. y cols.: "A model for the number of cores per prostate biopsy based on patient age and prostate gland volume”. J. Urol., 159: 920, 1998.

30. MATLAGA, B.R.; ESKEW, L.A.; McCULLOUGH, D.L.: "Prostate biopsy: indications and technic". J. Urol., 169: 12, 2003.

**31. VEIGA, F.G.; CASTELO, L.A.; SUAREZ, G. y cols.: "Technique of prostatic ultrasound guided biopsies with 10 samples. Is it useful?". Eur. Urol., 39: 182, 2001.

**32. CHOO, R.; KLOTZ, L.; DANJOUX, C. y cols.: "Feasibility study: watchful waiting for localized low to intermediate grade prostate carcinoma with selective delayed intervention based on prostate specific antigen, histological and/or clinical progression”. J. Urol., 167: 1664, 2002. 